\title{
Progresos en la detección y proyecciones de cambio climático en \\ España desde el informe 2010 de evaluación regional del clima pasado, presente y futuro del Comité CLIVAR-España
}

\section{Enrique Sánchez ${ }^{1}$, Belén Rodríguez-Fonseca ${ }^{2,3}$, Ileana Bladé ${ }^{4}$, Manola Brunet ${ }^{5}$, Roland Aznar ${ }^{6}$, Isabel Cacho ${ }^{7}$, María Jesús Casado ${ }^{8}$, Luis Gimeno", José Manuel Gutiérrez ${ }^{10}$, Gabriel Jordá ${ }^{11}$, Alicia Lavín ${ }^{12}$, José Antonio López ${ }^{8}$, Jordi Salat ${ }^{13}$, Blas Valero ${ }^{14}$}

\author{
${ }^{1}$ UCLM: Universidad de Castilla-La Mancha, Facultad CC Ambientales y Bioquímica, Toledo \\ ${ }^{2}$ UCM: Universidad Complutense de Madrid, Dept. Física de la Tierra y Astrofísica, Facultad CC Físicas, Madrid \\ ${ }^{3}$ IGEO: Instituto de Geociencias, UCM, Consejo Superior de Investigaciones Científicas, España \\ ${ }^{4}$ UB: Universitat de Barcelona, Dept. Física Aplicada, Facultat Física, Barcelona \\ ${ }^{5}$ URV-C3: Universitat Rovira i Virgili, Centre for Climate Change, Dept. Geografía, Tarragona \\ ${ }^{6}$ Puertos del Estado, Madrid \\ ${ }^{7}$ UB-GRC: Universitat de Barcelona, Geociències Marines, Dept. de Dinàmica de la Terra i de l'Oceà, \\ Facultat de Ciencies de la Terra, Barcelona \\ ${ }^{8}$ AEMET: Agencia Estatal de Meteorología, Madrid \\ ${ }^{9}$ UVIGO: Universidad de Vigo, Laboratorio de Física Ambiental, Vigo \\ ${ }^{10}$ UC-CSIC: Universidad de Cantabria, Grupo de Meteorología. Instituto de Física de Cantabria, Santander \\ ${ }^{11}$ UIB-IMEDEA: Universitat de les Illes Balears, Instituto Mediterráneo de Estudios Avanzados, \\ Palma de Mallorca \\ ${ }^{12}$ IEO: Instituto Español de Oceanografía, Centro Oceanográfico Santander \\ ${ }^{13}$ ICM-CSIC: Instituto de Ciencias Marinas, Barcelona \\ ${ }^{14}$ IPE-CSIC: Instituto Pirenaico de Ecología, Zaragoza
}

\begin{abstract}
Introducción
La península ibérica ofrece un marco apasionante para los estudios de variabilidad climática por varias razones. Tiene una amplia variedad de regímenes climáticos, que van desde condiciones atlánticas húmedas, con precipitaciones anuales de más de 2000 mm, a zonas amplias semiáridas, con estreses hidrológicos severos, e incluso climas fríos alpinos en algunas zonas aisladas. Esta diversidad climática es el resultado de su localización latitudinal en el borde norte de la franja subtropical, su compleja orografía salpicada por importantes cadenas montañosas, su naturaleza peninsular, y la presencia de dos masas de agua que la rodean con características muy diferentes: el océano Atlántico y el mar Mediterráneo. La variabilidad climática queda reflejada en importantes heterogeneidades en los balances de energía y humedad entre la superficie y la atmósfera, y los correspondientes intercambios. Además, eventos extremos como periodos prolongados sin lluvias, olas de calor, o precipitaciones convectivas severas e inundaciones son fenómenos recurrentes. Todos estos factores hacen que un entendimiento completo del clima ibérico, y su modelización sea un trabajo particularmente desafiante.
\end{abstract}

Como el resto de la región mediterránea, y como ha sido indicado en el último informe del IPCC de 2013 (WG1AR5,
Capítulo 14, Christensen et al., 2013), las proyecciones futuras sobre la PI indican que puede ser severamente afectada por importantes incrementos de temperatura y reducciones de precipitación, especialmente en verano, una alta probabilidad de mayor frecuencia de olas de calor. Esta perspectiva de importantes impactos negativos en una zona ya de por sí de alta vulnerabilidad refuerza la necesidad de una exhaustiva evaluación del clima actual de la región, para una mejor interpretación de las proyecciones climáticas, de forma que podamos mejorar nuestra fiabilidad sobre ellas.

La ciencia del clima se ha desarrollado más lentamente en España cuando se compara con otras regiones europeas, pero en la actualidad existe una gran comunidad nacional de investigadores en clima, involucrados en la evaluación del papel de los múltiples procesos relevantes, como la topografía, los modos de variabilidad, las teleconexiones con las zonas tropicales, las interacciones atmósfera-suelo y atmósfera-océano, para conocer mejor los diferentes climas regionales y entender mejor cómo pueden cambiar bajo las condiciones de cambio climático global. Este objetivo es posible por la existencia de series meteorológicas relativamente largas, y una densa red de estaciones, aunque por desgracia no todos los datos son accesibles de manera pública para los investigadores. 
Puesto que muchos de los estudios llevados a cabo por la comunidad científica de clima en España ajustan muy bien con los principales objetivos científicos del proyecto internacional CLIVAR (http://www.clivar.org/ about/about-clivar\#Objective), hace ya 15 años se creó una red de científicos, con el objetivo de coordinar la ciencia climática en España, y mejorar su visibilidad interna. Roberta Boscolo, en ese momento trabajando en la Oficina Internacional de CLIVAR, fue clave para promover este esfuerzo conjunto. Esto llevó a la creación de una red temática, que fue el embrión del actual Comité Científico CLIVAR-ESPAÑA (http://www.clivar.es/), que incluye representación de todos los diferentes campos relacionados con la ciencia de CLIVAR (meteorólogos, físicos de la atmósfera, oceanógrafos, paleocientíficos...). El Comité, sin casi soporte económico por parte del gobierno central, también se ha esforzado para servir de referencia para la comunidad científica del clima en España, y un punto de contacto con la sociedad y los políticos y legisladores, un enlace con el programa internacional CLIVAR.

Los primeros logros objetivos de este Comité fueron la organización de dos workshops nacionales, en 2005 y 2009, ambos seguidos de la publicación de informes de evaluación, disponibles en la web nacional de CLIVAR, y el segundo, en inglés también, en la web de CLIVAR internacional. El primero, titulado «Estado de la investigación del Clima en España», pretendía servir como una visión general de los grupos de investigación españoles que trabajaban en la ciencia del clima, el segundo, titulado «Clima en España: pasado, presente y futuro. Informe de evaluación del cambio climático regional» (Pérez y Boscolo, 2010) se planteó como un informe exhaustivo de evaluación del clima regional, con revisión por pares, en el que más de cien investigadores contribuyeron a su redacción. Fue el primer esfuerzo coordinado que involucró a toda la comunidad climática española en un empeño científico para mostrar, tanto nacional como internacionalmente su trabajo, como evidencia de la vitalidad y relevancia de la ciencia climática en España. Este trabajo fue presentado oralmente en la 18. ${ }^{a}$ sesión del comité de coordinación científica de CLIVAR en París (2011), y en la conferencia WCRP-OSC que tuvo lugar en Denver ese mismo año, a través de un conjunto de pósters.

Después de la publicación del informe IPCC 2013, el Comité CLIVAR-ESPAÑA pensó que era oportuno actualizar ese primer informe de evaluación. Con esa idea en mente, se llevó a cabo un simposio en Tortosa en 2015, titulado «Simposio internacional CLIMATE-ES 2015: Avances en la detección y proyecciones del cambio climático en España a la luz del 5. informe del IPCC-AR5» (http://www.climaes2015.urv.cat), con el soporte de varias instituciones de investigación, incluyendo AEMET. El principal objetivo de este simposio era servir de punto de partida para la actualización del informe de 2010. Sin embargo, debido a la falta de financiación institucional, se pensó que una opción más viable, y más efectiva sería presentar los resultados del simposio a través de un número especial de la revista CLIVAR Exchanges, que coordina la oficina internacional de CLIVAR (http://www.clivar.org/ publications/exchanges).
Los siguientes nueve artículos resumen los principales resultados presentados en el simposio de Tortosa. Cada artículo se concentra en uno de los aspectos del mismo, que fueron los siguientes: paleoclima, series temporales climáticas, bases de datos observacionales en malla, tendencias atmosféricas, teleconexiones, variabilidad oceánica observada, evaluación de la modelización climática regional tanto atmosférica como oceánica, y proyecciones climáticas regionales oceánicas (sobre el Atlántico y el Mediterráneo de manera diferenciada) y atmosféricas. La estructura es similar a la del primer informe, pero con un énfasis claro en la actualización de los resultados obtenidos en este periodo, avances en el entendimiento de procesos, y nuevos desarrollos, con el foco en nuevas investigaciones. El objetivo de esa publicación ha sido, pues, resaltar la amplitud de objetivos y estudios que lleva a cabo la comunidad científica nacional que en España trabaja en la ciencia del clima. Por ello, este volumen especial ha intentado incluir la mayor cantidad de grupos de investigación posibles, con la esperanza de que pueda servir de base para cualquier investigador español especializado en aspectos particulares.

Aunque el continuo crecimiento de la ciencia climática en España en estos últimos años es evidente, con un incremento constante de contribuciones en revistas internacionales y participación individual en proyectos internacionales e informes de IPCC, en el contexto no despreciable de la crisis económica y una reducción drástica de los presupuestos para ciencia de estos últimos años, el volumen especial pretendía servir como muestra de la capacidad de la comunidad climática española para trabajar junta y de manera coordinada, reconociendo que este volumen especial es solo una parte, inevitablemente incompleta, de todo el esfuerzo y logros de la comunidad nacional de estos últimos años.

Como comité nacional CLIVAR al mostrar este trabajo, queremos también agradecer y rendir tributo y reconocimiento a todos los investigadores que han contribuido directa o indirectamente a él, esperando que pueda ayudar a aumentar la visibilidad internacional y crear conciencia y reconocimiento sobre su trabajo. Por supuesto, también expresar gratitud a todos los miembros anteriores del comité, que con su ejemplo y entusiasmo abrieron camino y nos mostraron que iniciativas como esta son posibles incluso sin soporte institucional.

Lo que sigue es un breve resumen de los principales logros de cada uno de los artículos:

\section{Paleoclima}

Las reconstrucciones disponibles terrestres y marinas en la península ibérica en los últimos 2000 años muestran la evidencia de una compleja evolución espacial y temporal de la precipitación y temperatura.

Estos resultados replican de alguna manera la variabilidad climática actual en la península ibérica, con una compleja orografía, que da lugar a una gran presencia de microclimas. La evolución climática de la península ibérica en los últimos dos milenios puede ser dividida en cuatro periodos climáticos principales: el periodo Romano, la primera edad medieval, la anomalía climática medieval y la pequeña edad 
de hielo, caraterizadas por patrones distintivos de temperatura y precipitación. Un gran esfuerzo debe ser llevado a cabo para obtener reconstrucciones climáticas más robustas y multiproxies en áreas donde la cobertura geográfica es todavía vaja, como en el centro de la península ibérica y en el área marina del norte. Además, un mejor entendimiento de la evolución de los principales modos climáticos en escalas decadales a siglos, especialmente los conocidos como EA (Eastern Atlantic, Atlántico Este) o SCAND (Escandinavo) es de enorme importancia, así como las interacciones temporales entre ellas, y con la irradiancia solar total.

\section{2-3: Reconstrucciones instrumentales}

En los últimos años, se ha llevado a cabo un gran esfuerzo para mejorar la calidad de las herramientas y las observaciones atmosféricas, incluyendo bases de datos en malla, que se usan para analizar el sistema climático desde diferentes puntos de vista. En este sentido, diferentes iniciativas y proyectos se han llevado a cabo con el objetivo de rescatar y digitalizar los datos observacionales existentes, con especial atención a regiones con una pobre cobertura espacial y/o temporal, y desarrollar métodos y herramientas adecuados para elaborar bases de datos de alta calidad para el análisis climático. Diversos productos a escala regional y nacional en malla regular se han desarrollado en los últimos años con propósitos investigadores, cubriendo un amplio rango de aplicaciones, resoluciones, variables y periodos temporales. No obstante, la principal limitación que debe ser indicada es que la mayor parte de las bases de datos de alta calidad desarrolladas a escala local, regional o nacional en España son raramente compartidas en la comunidad climática, dando lugar a análisis redundantes en muchos casos. Por otro lado, no existen análisis de intercomparación adecuados entre las diferentes bases de datos desarrolladas.

\section{4-5: Tendencias. Variabilidad atmosférica}

Observaciones durante las últimas décadas revelan que hay un escenario más cálido y seco en comparación con las décadas anteriores, un descubrimiento que es compatible con las observaciones de otras áreas mediterráneas. En particular, ha habido un fuerte incremento en la radiación solar en la década de 1980, en acuerdo con un incremento de la demanda evaporativa atmosférica, especialmente en los meses de verano. Las temperaturas han mostrado fuertes incrementos desde la década de 1960. Sin embargo, la velocidad del viento en superficie no ha mostrado cambios perceptibles. Un fuerte descenso en la humedad relativa, sin cambios significativos identificables en la humedad absoluta. Aunque se han encontrado fuertes tendencias en la variabilidad espacial y estacional de la precipitación, puede estar en relación con cambios en las teleconexiones globales. La precipitación media anual sobre España ha mostado un descenso moderado en las últimas cinco décadas. En relación con las teleconexiones, se han llevado a cabo investigaciones relevantes en las últimas décadas relacionadas con la predictibilidad de la NAO (North Atlantic Oscillation, Oscilación del Atlántico Norte), mostrando cómo los trópicos y la estratosfera ayudan a mejorar dicha predictibilidad. Los escenarios de clima futuro proyectan un incremento de la NAO a lo largo del ciclo estacional, con impactos también en el nivel del mar y en los afloramientos (véanse artículos sobre regionalización oceánica en este volumen). No obstante, el patrón espacial de la NAO se ha visto que es no estacionario, y dependiente de la influencia de fondo que el ENSO (El Niño-Southern Oscillation, El Niño-Oscilación del Sur) y las variaciones lentas del océano ejercen sobre la variabilidad atmosférica. Los océanos Atlántico y Pacífico, y el Mediterráneo, la estratosfera, y la convección tropical se ha encontrado que modulan los patrones de teleconexión que afectan Europa. Teniendo en cuenta esta información, un enorme progreso se ha llevado a cabo en las décadas recientes en el desarrollo de aplicaciones para predicción en escalas entre estacionales y decadales (s2d) y entre subestacional y estacional (s2s).

La disponibilidad de resultados por parte del proyecto sobre predicción histórica del sistema climático (CHFP, Climate system Historical Forecasting), y el proyecto de intercomparación de modelos acoplados (CMIP5, Coupled Model Intercomparison Project), junto con las investigaciones muy activas en las comunidades tanto de grupos operativos como de investigadores, ha mejorado nuestas capacidades para obtener predicciones y proyecciones futuras de gran calidad y fiabilidad. El Centro de Supercomputación de Barcelona (BSC, Barcelona Supercomputing Center) y AEMET son las instituciones más importantes que lideran estos progresos, siendo un paso adelante muy significativo hecho por la comunidad investigadora española en el contexto de los servicios climáticos.

\section{6: Observaciones oceánicas}

Los resultados confirman los impactos del calentamiento global en el océano en las escalas regionales en torno a la península ibérica.

Este informe resalta la importancia de la monitorización regular de las zonas marinas. Por desgracia, muchas fuentes de financiación cuentan con una duración limitada, típicamente 3-4 años. También es cierto que muchos esfuerzos de monitorización en los que se basa este informe, y que actualmente están mantenidos por el IEO (Instituto Español de Oceanografía) y otras instituciones (Puertos, Universidades, etc.) se iniciaron a través de proyectos científicos específicos. Es el caso del sistema de observación IEO (IEO Observing system), y la estación de monitorización de Espartel. Otras estaciones de monitorización empezaron como iniciativas voluntarias a muy bajo coste, como por ejemplo, la red de Hidrocambios, o algunas de las estaciones más antiguas de registros costeros (Aquarium en San Sebastián, o la estación de L' Estartit). En todos los casos, su utilidad para seguir la evolución del cambio climático en los años pasados ha sido probada, así como el estudio de fuentes biológicas y su dependencia de variables fisico-químicas, todas ellas aspectos cruciales en la implementación de la estrategia marco marina de la UE. No obstante, una estrategia más coordinada que considere la capacidad global del sistema de observaciones para detectar señales de cambio climático sería muy bienvenida.

\section{7-8: Regionalización atmosférica: descripción de clima presente y proyecciones climáticas futuras}

Desde 2010 se han publicado múltiples estudios analizando el clima regional mediante modelos (RCMs) para condiciones de clima presente. Euro y Med-CORDEX o los proyectos ENSEMBLES y ESCENA (nacional) han sido las principales iniciativas durante este periodo. La 
principal conclusión de estos estudios es que los RCM sobre la península ibérica proporcionan una información muy valiosa, mostrando la distribución local espacial de variables climáticas, principalmente debido a una mejor representación de la orografía y los aspectos de la superficie. Los RCM son claramente capaces de capturar los regímenes de precipitación, temperatura y variabilidad del viento, así como los eventos extremos. No obstante, todavía se obtienen importantes sesgos, dificultando la aplicación directa de las salidas de los RCM en aplicaciones sectoriales (hidrología, agricultura, y energía, por ejemplo). Este aspecto abre un tema controvertido sobre la corrección o ajuste de sesgos de los RCM y su impacto en la señal de cambio climático. En relación con los procedimientos de regionalización estadística (SDM), estos métodos se han mostrado como una alternativa espacialmente consistente a los métodos estándar de corrección de sesgos, aunque la limitación para ser usados con valores extremos debe ser tenida en cuenta cuidadosamente en aplicaciones donde este aspecto sea relevante. Entre los beneficios de los SDM, puede ser resaltado el que son menos costosos computacionalmente que los RCM y permiten la regionalización de variables no meteorológicas.

Respecto a las proyecciones de cambio climático regional atmosférico, a partir del gran conjunto de metodos de regionalización mostrado en este volumen de Exchanges, y teniendo en cuenta las diversas incertidumbres indicadas, algunas conclusiones globales se pueden indicar. Para la precipitación, hay una tendencia general hacia el descenso en la precipitación promedio en todas las estacionales, con un descenso promedio del $30 \%$ según las estimaciones de los RCM. Respecto a la temperatura, los incrementos mayores se proyectan en verano y otoño, alcanzando cerca de $3{ }^{\circ} \mathrm{C}$ respecto a la climatología de 1971-2000 en el final del periodo del siglo XXI, y no menos de $1{ }^{\circ} \mathrm{C}$ en las estimaciones más conservadoras. Algunos aspectos relevantes deben ser mencionados para la aplicación del cambio climático regional en aplicaciones a estudios de impactos: diversos métodos de corrección/ajuste de sesgos se han desarrollado en estos años, y se muestran múltiples ejemplos de uso específico y sus limitaciones. Un foco principal de los estudios de proyecciones de cambio climático es la aplicación de la información relevante sobre cambio climático a la investigación sobre vulnerabilidad, impactos y adaptación. No obstante, no está claro todavía cuál es el mejor procedimiento para seleccionar un subconjunto de datos representativo para cada estudio particular, pues se ha visto que puede encontrarse información inconsistente e incluso contradictoria. Es este un aspecto clave a ser considerado en las iniciativas actuales en marcha en los grupos científicos de investigación en cambio climático.

\section{9: Regionalización oceánica: descripción de clima presente y proyecciones climáticas futuras}

Una pequeña reducción en la altura de la ola y su periodo medio se ha encontrado a lo largo de las costas atlánticas y mediterráneas. En el Atlántico noreste, el nivel del mar se prevé que se incremente a un ritmo mayor que el valor global medio, aunque la incertidumbre es grande. Se prevé un aumento de temperatura en el Atlántico en todos los modelos aunque la desaceleración del AMOC (Atlantic Meridional Overturning Circulation), también descrito en el capítulo de observaciones oceánicas, puede reducir la advección de calor hacia el noroeste, y modular dicho calentamiento. El aumento de la cantidad de aguas afloradas puede (al menos parcialmente) contrarrestar el calentamiento del mar abierto en una banda estrecha a lo largo de la costa occidental ibérica.

En cuanto a la salinidad, el aumento de los flujos de agua dulce en latitudes altas del Atlántico Norte y/o el aumento de la fusión del hielo en Groenlandia traería aguas más dulces hacia las costas del Atlántico Nororiental. Por otro lado, se espera que la pérdida de agua dulce aumente en el Mediterráneo induciendo un aumento en la salinidad de la cuenca, aunque la advección de aguas menos saladas provinientes del Atlántico puede contrarrestar parcialmente este proceso.

En cuanto a los eventos extremos, está claro que el impacto de las futuras tormentas marinas aumentará debido al aumento del nivel medio del mar, pero no está claro si el número e intensidad de tormentas cambiará en el futuro. Algunos resultados apuntan a una disminución en el número de tormentas pero también a un aumento de los eventos más intensos, aunque la significación estadística de esos resultados es débil. El último informe del Grupo Intergubernamental de Expertos sobre el Cambio Climático (IPCC) afirma que existe una gran incertidumbre asociada con vientos y tormentas futuras.

En cuanto a las fuentes de incertidumbre de las proyecciones regionales, el factor más determinante en general es el GCM utilizado para forzar los modelos regionales (atmosféricos y oceánicos). En cuanto al escenario de emisión, los resultados apuntan a mostrar cambios más fuertes en los escenarios más pesimistas. Esto está claro para el nivel del mar y la temperatura. Sin embargo, para la salinidad y el oleaje, la relación no es tan robusta. 